\title{
Atividade Antibacteriana, Antioxidante e Compostos Fenólicos de Méis Produzidos por Nannotrigona testaceicornis Lepeletier (Apidae, Meliponini)
}

\author{
Antibacterial Activity, Antioxidant and Phenolic Compounds of Honeys Produced by Nannotrigona \\ testaceicornis Lepeletier (Apidae, Meliponini) \\ Atividade Antibacteriana, Antioxidante y Compostos Fenólicos de Méis Produzidos por \\ Nannotrigona testaceicornis Lepeletierta (Apidae, Meliponini)
}

\author{
Érica Larissa Silva Carvalho \\ ORCID: https://orcid.org/0000-0002-8642-6672 \\ Universidade do Estado da Bahia, Brasil \\ E-mail: erica.lari8@gmail.com \\ Ellen Matos Silva Bomfim \\ ORCID: https://orcid.org/0000-0002-8487-2484 \\ Universidade do Estado da Bahia, Brasil \\ E-mail: ellen.matos1@gmail.com \\ Marcos da Costa Silva \\ ORCID: https://orcid.org/0000-0002-3809-0297 \\ Universidade do Estado da Bahia, Brasil \\ E-mail: marcosilva@uneb.br \\ Luciene Cristina Lima e Lima \\ ORCID: https://orcid.org/0000-0003-1541-2991 \\ Universidade do Estado da Bahia, Brasil \\ E-mail: 1clima@uneb.br \\ Edson de Jesus Marques \\ ORCID: https://orcid.org/0000-0001-8371-6706 \\ Universidade do Estado da Bahia, Brasil \\ E-mail: emarques@uneb.br \\ Vera Lúcia Costa Vale \\ ORCID: https://orcid.org/0000-0002-4055-5049 \\ Universidade do Estado da Bahia, Brasil \\ E-mail: vvale@uneb.br
}

\begin{abstract}
Resumo
O mel de abelhas vem sendo utilizado na medicina popular para o tratamento de infecções e contra danos oxidativos. Entretanto, a composição do mel é bastante variável, pois depende da composição do néctar da espécie vegetal, da espécie de abelha produtora, das condições ambientais, da coleta e processamento. O presente estudo objetivou avaliar a atividade antibacteriana, antioxidante, teor dos compostos fenólicos totais e flavonoides totais do mel de Nannotrigona testaceicornis L. A atividade antibacteriana foi realizada pelo método de difusão em discos e concentração inibitória mínima. A capacidade antioxidante foi investigada através do ensaio de captura do radical livre 2,2-difenil-1-picrilhidrazila. O teor total de fenóis totais e flavonoides totais foi quantificado por espectrofotometria. As amostras dos meses de maio e junho/2013 inibiram o crescimento bacteriano para todas as bactérias analisadas. Todas as amostras de mel apresentaram ação antibacteriana frente a Pseudomonas aeruginosa. $\mathrm{O}$ teste ANOVA mostrou que houve diferença estatisticamente significante entre os meses para as bactérias Pseudomonas aeruginosa; Escherichia coli; Micrococcus luteus e Staphylococcus aureus. No ensaio de determinação da concentração inibitória mínima, as amostras de maio/2013, março/2014 e junho/2014 foram mais ativas frente a Micrococcus luteus. A capacidade antioxidante acima de $60 \%$ foi observada na amostra de maio/2013. As amostras de janeiro/2014 e maio/2103 se destacaram na quantificação de fenóis totais e flavonoides totais, respectivamente. Houve correlação positiva entre a ação antioxidante e flavonoides totais. $\mathrm{O}$ mel in natura de Nannotrigona testaceicornis L. apresenta potencial antimicrobiano e capacidade sequestradora de radicais livres.
\end{abstract}

Palavras-chave: Antimicrobiano; Abelha nativa; Fenóis totais; DPPH.

\begin{abstract}
Bee honey is used in folk medicine to treat infections and oxidative damage. The composition of honey is quite variable, as it depends on the type of nectar of the plant species, the species of bee that produces it, the environmental conditions, collection and processing. This study aimed to evaluate the antibacterial and antioxidant activity, the
\end{abstract}


content of total phenolic compounds and total flavonoids in honey from Nannotrigona testaceicornis L. The antibacterial activity was performed by the disk diffusion method and by the minimum inhibitory concentration. The antioxidant capacity was evaluated using the 2,2-diphenyl-1-picrylhydrazyl free radical scavenging assay. The content of total phenols and flavonoids was quantified by spectrophotometry. It was observed that honey samples from May and June/2013 inhibited bacterial growth for all analyzed bacteria (Pseudomonas aeruginosa, Escherichia coli; Micrococcus luteus and Staphylococcus aureus). The ANOVA test showed that there was a statistically significant difference between months for P. aeruginosa; E. coli; M. luteus and S. aureus. In the assay to determine the minimum inhibitory concentration, samples from May/2013, March/2014 and June/2014 were more active against M. luteus. Antioxidant capacity above $60 \%$ was observed in the sample from May/2013. The samples from January/2014 and May/2103 stood out in the quantification of total phenols and total flavonoids, respectively. There was a positive correlation between antioxidant action and total flavonoids. Honey in natura from Nannotrigona testaceicornis L. showed antimicrobial potential and free radical scavenging capacity.

Keywords: Antimicrobial; Native bee; Total phenols; DPPH.

\section{Resumen}

La miel de abeja se usa en la medicina popular para tratar infecciones y daño oxidativo. La composición de la miel es bastante variable, ya que depende del tipo de néctar de la especie vegetal, la especie de abeja que la produce, las condiciones ambientales, recolección y procesamiento. Este estudio tuvo como objetivo evaluar la actividad antibacteriana y antioxidante, el contenido de compuestos fenólicos totales y flavonoides totales en miel de Nannotrigona testaceicornis L. La actividad antibacteriana se realizó por el método de difusión en disco y por la concentración mínima inhibitoria. La capacidad antioxidante se evaluó utilizando el ensayo de eliminación de radicales libres 2,2-difenil-1-picrilhidrazilo. El contenido de fenoles y flavonoides totales se cuantificó mediante espectrofotometría. Se observó que las muestras de miel de mayo y junio de 2013 inhibieron el crecimiento bacteriano de todas las bacterias analizadas (Pseudomonas aeruginosa, Escherichia coli; Micrococcus luteus y Staphylococcus aureus). La prueba ANOVA mostró que hubo una diferencia estadísticamente significativa entre meses para $P$. aeruginosa; E. coli; M. luteus y S. aureus. En el ensayo para determinar la concentración mínima inhibitoria, las muestras de mayo / 2013, marzo / 2014 y junio / 2014 fueron más activas contra M. luteus. En la muestra de mayo de 2013 se observó una capacidad antioxidante superior al 60\%. Las muestras de enero / 2014 y mayo / 2103 se destacaron en la cuantificación de fenoles totales y flavonoides totales, respectivamente. Hubo una correlación positiva entre la acción antioxidante y los flavonoides totales. La miel in natura de Nannotrigona testaceicornis L. mostró potencial antimicrobiano y capacidad de captación de radicales libres.

Palabras clave: Antimicrobiano; Abeja nativa; Fenoles totales; DPPH.

\section{Introdução}

O mel é um alimento de alta qualidade nutricional e com elevado potencial terapêutico, graças as suas características físico-químicas. Tem sido utilizado como antimicrobiano desde os tempos mais remotos, antes mesmo que as bactérias fossem descobertas como responsáveis por infecções (Molan, 2001; Amèndola, 2003). A utilização do mel na medicina tradicional está relacionada ao tratamento de anemias, úlceras estomacais, feridas, queimaduras, problemas oftalmológicos e digestivos (Verissimo, 1987).

Propriedades antimicrobianas, antioxidantes, anti-inflamatórias, anticâncer e cicatrizantes do mel foram relatados na literatura (Bueno-Costa et al., 2016; Albaridi, 2019). A atividade terapêutica do mel está relacionada a presença dos compostos polifenólicos, carotenoides, aminoácidos, enzimas, proteínas, vitaminas e minerais (Chua et al., 2013). A diversidade da composição bioquímica do mel é procedente das múltiplas fontes florais utilizadas para a coleta do néctar, dos fatores sazonais e ambientais, bem como o processamento (Kivrak \& Kivrak, 2017).

Dentre as propriedades farmacológicas do mel, a atividade antibacteriana se destaca devido ao cenário atual do uso excessivo e indiscriminado de antibióticos tanto na agricultura quanto na saúde humana, impulsionando a resistência bacteriana e a necessidade de medicamentos com novos mecanismos de ação (Gonçalves et al., 2005). Os compostos bioativos do mel podem contribuir para o desenvolvimento e obtenção de novos fármacos. Segundo os estudos de Olaitan et al. (2007) e Aggad e Guemour (2014), o mel apresenta ação bactericida e bacteriostática frente a diversos microrganismos, como Staphylococcus aureus, Escherichia coli e Pseudomonas spp. 
Os meliponíneos constituem um grupo de abelhas formado por mais de 300 espécies conhecidas em todo o mundo, sendo encontradas nas regiões tropicais e subtropicais da Terra (Freitas, 2003). No Brasil, os meliponíneos são encontrados em todas as regiões, com 27 gêneros e mais de 250 espécies (Silveira et al., 2002; Alves et al., 2007). Entre os Meliponíneos, a espécie Nannotrigona testaceicornis (Lepeletier) possui grande distribuição geográfica, abrangendo as regiões do Nordeste até o Sul do Brasil (Michener, 1974; Silveira et al., 2002), tem fácil adaptação a regiões antrópicas e de um modo geral não é agressiva (Rodrigues, 2006).

Devido às particularidades das produções das abelhas sem ferrão, especialmente do mel, cresce a motivação de diversos estudos com a finalidade de avaliar as atividades farmacológicas e as características físico-químicas desses méis. No Brasil, apesar da grande extensão territorial e da variedade florística e climática, trabalhos como estes ainda são escassos. É evidente a necessidade de estudos científicos que visem caracterizar e identificar o potencial biotecnológico dos produtos das abelhas nativas, colocando em destaque o potencial destes méis do estado da Bahia para posteriores estudos no campo medicinal, nutricional e biotecnológico, ampliando o respaldo da qualidade do mel de Nannotrigona testaceicornis L.

O objetivo do presente estudo foi avaliar a atividade antibacteriana, antioxidante e quantificar os fenóis totais e flavonoides totais dos méis da abelha Nannotrigona testaceicornis L.

\section{Metodologia}

O presente estudo é uma pesquisa experimental com abordagem metodológica quantitativa, realizada por coleta de dados através de técnicas matemáticas (Pereira et al., 2018), para fins de avaliação da atividade antibacteriana, antioxidante e quantificação dos compostos fenólicos totais e flavonoides totais dos méis da Nannotrigona testaceicornis L.

\subsection{Mel}

As amostras de mel não pasteurizado de Nannotrigona testaceicornis L. foram coletadas entre maio de 2013 e março de 2014 no meliponário da Universidade do Estado da Bahia, no município de Alagoinhas, Bahia, Brasil (1208'08” S / $38^{\circ} 25^{\prime} 09^{\prime \prime}$ W). Em 2013 foram coletadas amostras nos meses de maio, junho, agosto e novembro. Em 2014 foram coletadas as amostras nos meses de janeiro, fevereiro e março. As amostras foram envasadas em tubos estéreis Falcon e armazenados no escuro à temperatura de $4^{\circ} \mathrm{C}$.

\subsubsection{Avaliação da atividade antibacteriana in vitro}

A avaliação antimicrobiana foi determinada pela técnica de disco-difusão em agar, de acordo com a metodologia descrita do CLSI - Clinical \& Laboratory Standards Institute (2003), com bactérias Bacillus subillis (ATCC 6633), Micrococus luteus (ATCC 10240), Staphylococcus aureus (ATCC 25923), Pseudomonas aeruginosa (ATCC 15442) e Escherichia coli (ATCC 94863).

Discos de papel de filtro estéreis com $6 \mathrm{~mm}$ de diâmetro impregnados com $100 \mu \mathrm{g}$ de mel foram adicionados sobre a superfície do meio agar Mueller-Hinton inoculado com microrganismo-teste (106 células / mL) (Bauer et al., 1966, com adaptações). Como controle negativo foi utilizado discos acrescidos com solução salina a 0,9 \% e como controle positivo, discos com cloranfenicol $(0,1 \%)$. As placas foram incubadas em estufa a $37^{\circ} \mathrm{C}$ por 24 horas. A leitura dos resultados foi realizada medindo-se os halos de inibição de crescimento. $\mathrm{O}$ teste foi realizado em triplicata e com duas repetições.

\subsubsection{Avaliação da Concentração Inibitória Mínima (CIM)}

A Concentração Inibitória Mínima (CIM) foi realizada conforme as recomendações do documento M7-A6 do CLSI - 
Clinical \& Laboratory Standards Institute (CLSI, 2003), com modificações. Os microrganismos foram cultivados em placas de 96 poços com diferentes concentrações do mel (250 a 1,82 $\mu \mathrm{g} / \mathrm{mL})$. Foi utilizado Clorafenicol $(0,1 \%)$ como controle positivo e solução salina $(0,9 \%)$ como controle negativo. As placas foram incubadas por 24 horas a $37^{\circ} \mathrm{C}$. A CIM foi determinada como a menor concentração do extrato em $\mu \mathrm{g} / \mathrm{mL}$ que inibiu o crescimento microbiano. O teste foi realizado em triplicata e com duas repetições

\subsubsection{Avaliação in vitro da atividade antioxidante}

Para a avaliação da atividade antioxidante das amostras de mel foi utilizado o método colorimétrico do ensaio de captura do radical livre 2,2-difenil-1-picrilhidrazila (DPPH), em placas de 96 poços, adaptado (Brand-Willians et al., 1995). Os méis foram diluídos em solução salina a $0,9 \%$ obtendo concentrações finais de 500 a $3,90 \mathrm{mg} / \mathrm{mL}$. As leituras das absorbâncias foram realizadas em espectrofotômetro UV-Vis a $492 \mathrm{~nm}$. O teste foi procedido em triplicata e com duas repetições. Determinou-se a porcentagem de sequestro de radicais livres (\% SRL) através da equação: \% SRL = $\{[($ Abs controle - (Abs amostra - Abs branco)] x 100\} /Abs controle \} Onde: Abs controle - absorbância do DPPH e etanol. Abs amostra - absorbância do extrato após reação com DPPH. Abs branco - absorbância do etanol (Moreira et al., 2005).

\subsubsection{Quantificação de fenóis totais e flavonoides totais}

Compostos fenólicos totais foram quantificados pelo método espectrofotométrico utilizando a reação de FolinCiocalteu, com adaptações (Singleton et al., 1999). As amostras dos méis foram diluídas em diferentes concentrações (250 a 3,90 mg / mL). As placas foram armazenadas na ausência de luz por 40 min e a absorbância foi lida em espectrofotômetro a $620 \mathrm{~nm}$. Os resultados foram expressos em equivalentes de ácido gálico por grama de mel (mg EAG / g), através da interpolação das absorbâncias das amostras com a curva padrão de ácido gálico.

A quantificação de flavonóides totais foi analisada pelo método descrito por Arvouet-Grand et al. (1994) em placa de poliestireno com 96 poços, em triplicata. As absorbâncias das reações colorimétricas resultantes, foram lidas em espectrofotômetro a $492 \mathrm{~nm}$. O conteúdo de flavonoides foi expresso em equivalentes de quercetina (mg EQ / 100 g) calculados através de curva padrão. Os testes foram procedido em triplicata e com duas repetições

\subsection{Análise estatística}

Os dados foram expressos em valores de média e desvio padrão utilizando o GraphPadPrism 5.0. Em cada parâmetro as diferenças entre as amostras (mês) foram analisadas utilizando Análise de Variância Unidirecional (ANOVA) seguida pela múltipla comparação mês a mês HSD de Tukey. Valores de p menores ou iguais a 0,05 foram avaliados estatisticamente significativo. Foram calculados os coeficientes de correlação de Pearson entre as covariáveis para os compostos fenólicos, flavonóides e para atividade antioxidante. Estes tratamentos foram realizados utilizando o programa Statistical Package for The Social Sciences 21 (SPSS 21).

\section{Resultados}

As amostras do mel da abelha sem ferrão Nannotrigona testaceicornis L. possibilitou a formação de halos de inibição frente aos microrganismos testados. Os méis coletados nos meses de maio, junho e novembro de 2013 foram ativos contra todos as espécies de bactérias conforme a Tabela 1. 
Tabela 1. Halo de inibição $(\mathrm{mm})$ da atividade de méis de Nannotrigona testaceicornis L. pela técnica de disco-difusão em ágar.

\begin{tabular}{cccccc}
\hline Amostras & $\begin{array}{c}\text { Escherichia } \\
\text { coli }\end{array}$ & $\begin{array}{c}\text { Micrococus } \\
\text { luteus }\end{array}$ & $\begin{array}{c}\text { Staphylococcus } \\
\text { aureus }\end{array}$ & $\begin{array}{c}\text { Pseudomonas } \\
\text { aeruginosa }\end{array}$ & $\begin{array}{c}\text { Bacillus } \\
\text { subtilis }\end{array}$ \\
\hline Maio/2013 & $9,0 \pm 1,0^{\mathbf{a}}$ & $8,3 \pm 1,15^{\mathbf{a}}$ & $8,3 \pm 0,57^{\mathbf{a}}$ & $12,0 \pm 1,0^{\mathbf{a}}$ & $7,3 \pm 0,57$ \\
Junho/2013 & $7,6 \pm 0,57^{\mathbf{a}}$ & $7,6 \pm 0,57$ & $10,3 \pm 1,15^{\mathbf{a b}}$ & $12,3 \pm 1,15^{\mathbf{b}}$ & $8,3 \pm 1,52$ \\
Agosto/2013 & - & $6,6 \pm 0,57^{\mathbf{a}}$ & $10,0 \pm 0,0^{\mathbf{c}}$ & $6,6 \pm 0,57^{\mathbf{a b c}}$ & - \\
Novembro/2013 & $7,0 \pm 1,0^{\mathbf{a}}$ & $7,6 \pm 0,57$ & $7,0 \pm 0,0^{\text {abc }}$ & $8,6 \pm 0,57^{\mathbf{a b c}}$ & $7,0 \pm 0,0$ \\
Janeiro/2014 & - & $6,6 \pm 0,57^{\mathbf{a}}$ & $7,0 \pm 0,0^{\mathbf{b c}}$ & $8,6 \pm 0,57^{\text {abc }}$ & - \\
Fevereiro/2014 & $8,0 \pm 0,0^{\mathbf{a}}$ & $6,6 \pm 0,57^{\mathbf{a}}$ & - & $8,3 \pm 0,57^{\mathbf{a b c d}}$ & - \\
Março/2014 & $7,5 \pm 0,7$ & - & $7,5 \pm 0,7^{\mathbf{b c}}$ & $10 \pm 1,73^{\text {abcd }}$ & $7,5 \pm 0,7$ \\
\hline Controle & $13 \pm 1,52$ & $14 \pm 0,57$ & $13 \pm 0,57$ & $12 \pm 1,52$ & $18 \pm 1,15$ \\
\hline
\end{tabular}

(-): Ausência de halo de inibição; Controle bacteriano: Cloranfenicol. Cada valor representa a média \pm desvio padrão das análises. Comparações entre os meses do mel de Nannotrigona testaceicornis L. realizada por ANOVA seguida do teste de Tukey. Diferenças estatisticamente significativas indicadas por letras diferentes nas colunas ( $\mathrm{p}<0,05)$. Fonte: Autores.

Conforme a Tabela 2, as amostras de maio/2013, março/2014 e junho/2014 foram mais ativas frente a M. luteus, com CIM de 7,81 $\mu \mathrm{g} / \mathrm{mL}$. A menor concentração para S. aureus ocorreu em junho/2013 e para B. subtilis em maio/2013 e março2014. As amostras de méis não apresentaram atividade frente às bactérias Gram-negativas, E. coli e P. aeruginosa nas concentrações testadas.

Tabela 2. Concentração Inibitória Mínima $(\mu \mathrm{g} / \mathrm{mL})$ das amostras de méis de Nannotrigona testaceicornis L.

\begin{tabular}{|c|c|c|c|c|c|}
\hline Amostras & $\begin{array}{c}\text { Escherichia } \\
\text { coli }\end{array}$ & $\begin{array}{c}\text { Micrococus } \\
\text { luteus }\end{array}$ & $\begin{array}{c}\text { Staphylococcus } \\
\text { aureus }\end{array}$ & $\begin{array}{c}\text { Pseudomonas } \\
\text { aeruginosa }\end{array}$ & $\begin{array}{c}\text { Bacillus } \\
\text { subtilis }\end{array}$ \\
\hline Maio/2013 & - & 7,81 & 31,25 & - & 15,62 \\
\hline Junho/2013 & - & 7,81 & 15,62 & - & - \\
\hline Agosto/2013 & NT & NT & NT & NT & NT \\
\hline Novembro/2013 & - & 15,62 & - & - & 31,25 \\
\hline Janeiro/2014 & - & 31,25 & - & - & - \\
\hline Fevereiro/2014 & - & - & - & - & - \\
\hline Março/2014 & - & 7,81 & - & - & 15,62 \\
\hline Controle & 0,03125 & 0,01562 & 0,03125 & 0,03125 & 0,01562 \\
\hline
\end{tabular}

(-): Ausência de inibição NT: Não testada. Fonte: Autores.

A quantificação de fenóis totais, flavonóides totais e a capacidade sequestrante de DPPH das amostras de méis de Nannotrigona testaceicornis L. encontram-se na Tabela 3. Os teores de fenóis variaram de 23,92 $\pm 2,89$ a 98,67 $\pm 12,45 \mathrm{mg}$ EAG. $\mathrm{g}^{-1}$. Os meses com maiores teores de fenóis totais foram janeiro $\left(91,08 \pm 22,31 \mathrm{mg}\right.$ EAG. $\left.\mathrm{g}^{-1}\right)$, fevereiro $(86,46 \pm 8,06 \mathrm{mg}$ EAG. $\left.\mathrm{g}^{-1}\right)$ e março $\left(98,67 \pm 12,45 \mathrm{mg}\right.$ EAG. $\left.\mathrm{g}^{-1}\right)$. Os teores de flavonoides totais variaram entre 7,3 \pm 0,33 a 27,46 $\pm 0,91$, com o melhor resultado os para os meses de maio/2013 (27,46 $\pm 0,91 \mathrm{mg}$ EQ. $\left.\mathrm{g}^{-1}\right)$ e junho/2013 (25,21 $\pm 0,96 \mathrm{mg}$ EQ. $\left.\mathrm{g}^{-1}\right)$.

Para a atividade sequestradora de radicais livres (DPPH), a amostra de mel de maio/2013 foi mais expressiva (63,21 $\% \pm 4,28)$. Os resultados variaram entre $25,54 \% \pm 5,83$ a $63,21 \% \pm 4,28$. 
Tabela 3. Quantificação de fenóis totais, flavonóides totais e porcentagem de sequestro de radicais livres (\% SRL) in vitro das amostras de méis de Nannotrigona testaceicornis L.

\begin{tabular}{|c|c|c|c|}
\hline Amostra & $\begin{array}{l}\text { Fenóis totais } \\
\left(\mathrm{mg} \text { EAG. } \mathrm{g}^{-1}\right)\end{array}$ & $\begin{array}{l}\text { Flavonóides totais } \\
\quad\left(\mathrm{mg} \mathrm{EQ} \cdot \mathrm{g}^{-1}\right)\end{array}$ & $\begin{array}{c}\text { DPPH } \\
(\% \text { SRL) }\end{array}$ \\
\hline Maio/2013 & $33,49 \pm 4,95^{\mathrm{a}}$ & $27,46 \pm 0,91^{\mathbf{a}}$ & $63,21 \pm 4,28^{\mathbf{a}}$ \\
\hline Junho/2013 & $23,92 \pm 2,89^{\mathbf{b}}$ & $25,21 \pm 0,96^{\mathbf{a b}}$ & $55,46 \pm 4,94^{\mathbf{b}}$ \\
\hline Agosto/2013 & $31,68 \pm 7,85^{\mathrm{c}}$ & $15,85 \pm 0,66^{\mathbf{a b c}}$ & $51,80 \pm 2,32^{a c}$ \\
\hline Novembro/2013 & $39,27 \pm 9,96^{\mathbf{d}}$ & $13,50 \pm 0,80^{\text {abcd }}$ & $55,46 \pm 0,49^{d}$ \\
\hline Janeiro/2014 & $91,08 \pm 22,31^{\text {abcd }}$ & $9,67 \pm 1,19^{\text {abcde }}$ & $55,37 \pm 7,73^{\mathrm{e}}$ \\
\hline Fevereiro/2014 & $86,46 \pm 8,06^{\text {abcd }}$ & NT & $25,54 \pm 5,83^{a b c}$ \\
\hline Março/2014 & $98,67 \pm 12,45^{\text {abcd }}$ & $7,30 \pm 0,33^{\text {abcde }}$ & $26,44 \pm 12,14^{\text {abcde }}$ \\
\hline Média & $57,79 \pm 9,78$ & $16,51 \pm 4,87$ & $47,61 \pm 5,39$ \\
\hline
\end{tabular}

NT: Não testada. Cada valor representa a média \pm desvio padrão das análises. Comparações entre os meses do mel de Nannotrigona testaceicornis L. realizada por ANOVA seguida do teste de Tukey. Diferenças estatisticamente significativas indicadas por letras diferentes nas colunas $(\mathrm{p}<0,05)$. Fonte: Autores.

Levando em consideração a análise estatística dos resultados da atividade antioxidante, quantificação de fenóis totais e flavonóides totais das amostras dos méis in natura nas concentrações testadas, houve diferença estatisticamente significante com $\mathrm{p}<0,05$, entre os meses nas três condições (ANOVA). A análise de correlação entre as covariáveis antioxidante, fenol e flavonoides, durante os meses analisados, através do Coeficiente de Correlação de Spearman, mostrou resultado positiva somente entre a atividade Antioxidante e o conteúdo de Flavonóides, com p < 0,05 e $\mathrm{r}_{\mathrm{s}}=0,71$.

\section{Discussão}

Muitas pesquisas comprovam a eficácia dos efeitos medicinais atribuídos ao mel da abelha Apis melifera (Miorin et al., 2003; Cruz et al., 2014) No entanto, os estudos sobre o potencial dos produtos das abelhas sem ferrão, ainda são poucos.

Os dados obtidos neste trabalho demonstram que o mel Nannotrigona testaceicornis L. têm potencial para uso terapêutico no controle e combate de infecções microbianas causadas por microrganismos Gram negativos $E$. coli e $P$. aeruginosa e microrganismos Gram positivos, S. aureus, B. subtilis e M. luteus. Os microrganismos E. coli e P. aeruginosa por serem Gram negativos são muito mais difíceis de serem combatidos.

Pseudomonas aeruginosas são patógenos oportunistas para indivíduos imunocomprometidos (Kollef, 2013). Pseudomonas aeruginosas, Staphylococcus aureus e E. coli, são responsáveis pela maioria dos casos de pneumonia resultante de infecções hospitalares (Gima, et al 2020). M. lutteus não é um patógeno humano, entretanto permite o estabelecimento de patógenos como S. aureus (Boldock, et al, 2018). B. subtilis é um microrganismo formador de esporos e importantes contaminantes dos alimentos (Vaerewijck et al., 2001).

Gonçalves et al. (2005), obtiveram resultados semelhantes aos dados apresentados nesta pesquisa ao analisarem o mel de Nannotrigona testaceicornis L., coletados no estado de São Paulo. Bozoni (2012), comparando abelhas nativas, Nannotrigona testaceicornis L., Plebeia remota, Tetragona clavipes, Scaptotrigona depilis com Apis mellifera observou que os méis das abelhas nativas foram significativamente mais eficiente para a atividade antimicrobiana frente a E. coli, Klebisiella pneumoniae, S. aureus, P.aeruginosa, e para o fungo Trichophyton rubron, quando comparado a atividade de Apis mellifera para as bactérias.

O teor de compostos fenólicos desta pesquisa variou significativamente entre os meses avaliados. Trabalho realizado por Lira et al., (2014), com méis de Apis melifera e meliponineos observaram que a produção de fenóis totais obtidas foram em 
menores concentrações para Apis melifera quando comparadas aos meliponineos, Tetragonisca angustula e Scaptotrigona sp., entretanto, as análises dos méis foram em estados e em períodos diferentes.

Esse trabalho mostrou maior teor de flavonoides quando comparado a méis de abelhas africanizadas realizados por Lira et al.(2014).

Lira et al., (2014), obteve uma variação da atividade antioxidante entre 20,49 a 36,16 mg $\mathrm{mL}^{-1}$ para os méis de meliponíneos, valores estes menores do que o encontrado neste trabalho. Oliveira et al., (2012), trabalhando com méis de abelhas nativas Melipona fasciculata, M. flavolineata e a africanizada Apis mellifera, observaram que, os méis analisados apresentaram atividade antioxidante, principalmente os méis mais escuros que possuíram também teores mais elevados de polifenóis totais. Os resultados aqui apresentados mostram correlação positiva entre flavonoide e a capacidade sequestradora de radicais livres. Segundo Silva, et al. 2010, os flavonoides estão entre os principais compostos do metabolismo secundário com ação antioxidante.

Nossos resultados mostram que os meses com melhor atividade antibacteriana foram maio e junho de 2013 e novembro de 2014 tanto para o teste difusão em disco quanto a concentração mínima inibitória e atividade antioxidante. A atividade antimicrobiana e atividade antioxidante estão relacionados entre outros fatores aos constituintes químicos oriundos das espécies botânicas, pois, segundo Chanchao (2009) e Al-mamary et al., (2002), a constituição química do mel está ligada à sua origem botânica e geográfica e aos compostos do metabolismo secundário das plantas .

As diferenças na ação biológica do mel de Nannotrigona testaceicornis L., encontradas neste trabalho, são advindas do período de coleta, sendo influenciado diretamente pelo tipo botânico. Pois as diferentes espécies vegetais têm produtos resultantes do metabolismo secundário diferenciados em concentrações que irão repercutir na quantificação de compostos fenólicos, flavonoides e consequentemente na atividade antioxidante e antibacteriana.

\section{Conclusão}

Os dados obtidos neste trabalho demonstram que o mel de Nannotrigona testaceicornis L. têm potencial para o controle e combate de infecções microbianas e na neutralização de radicais livres. Portanto, sugere-se a realização de novos estudos químico-farmacológicos para determinar a efetividade do mel na atividade antioxidante, no controle microbiológico e o seu potencial biotecnológico. E assim acredita-se que o uso desse produto biológico possa estimular a prática da meliponicultura e trazer benefícios ao pequeno produtor com a comercialização, bem como, estimular ações para preservação de áreas florestais.

\section{Referências}

Aggad, H. \& Guemour, D. (2014). Honey Antibacterial Activity. Medicinal and Aromatic Plants Research Journals, 3: 2. 10.4172/2167-0412.1000152.

Albaridi, N. A. (2019). Antibacterial Potency of Honey. International Journal of Microbiology, 2019: 1-10. 10.1155/2019/2464507.

Al-mamary, M., Al-meeri, A. \& Al-habori, M. (2002) Antioxidant activities and total phenolics of different types of Honey. Nutrition Research, 22:9 10411047. 10.1016/S0271-5317(02)00406-2.

Alves, R. M. O., Souza, B. A., Sodre, G. S. \& Fonseca, A. A. O. (2007). Desumidificação: uma alternativa para a conservação do mel de abelhas sem ferrão. Mensagem Doce 91: 2-8. https://www.apacame.org.br/mensagemdoce/91/artigo.htm.

Arvouet-Grand, A., Vennat, B., Pourrat, A. \& Legret, P. (1994). Standardisation d'un extrait de propolis et identification des principaux constituants. Journal de Pharmacie de Belgique, 49: 462-468.

Bauer, A. W., Kirby, W. M. M., Sherris, J. C. \& Turk, M. (1966). Antibiotic susceptibility testing by a standardized single disk metodo. American Journal of Clinical Pathology, 45: 493-496.

Boldock, E., Surewaard, B. G. J., Shamarina, D., Na, M., Fei, Y., Ali, A., Williams, A., Pollitt, E. J. G., Szkuta, P., Morris, P., Prajsnar, T. K., McCoy, K. D., Jin,T., Dockrell, D. H., van Strijp, J. A. G., Kubes, P., Renshaw, S. A. \& Foster, S. J. (2018). Human skin commensals augment Staphylococcus aureus pathogenesis. Nature Microbiology, 3: 881-890. 10.1038/s41564-018-0198-3. 
Bazoni, M. O. 2012. Atividade antimicrobiana do mel produzido por Apis mellifera e abelhas sem ferrão nativas do Brasil. (2012). 116f. Tese (Doutorado em Ciências: Área de Concentração em Genética) - Faculdade de Medicina de Ribeirão Preto, Universidade de São Paulo, Ribeirão Preto.

Brand-Willians, W., Curvelier, M. E. \& Berset, C. (1995). Use of afree radical method to evaluate antioxidant activity. Food Science and Technology, 28:2530. 10.1016/S0023-6438(95)80008-5.

Bueno-Costa, F.M., Rui, C.Z., Bohmer, B.W., Chaves, F.C., Silva, W.P., Zanusso, J.T. \& Dutra, I. (2016). Antibacterial and antioxidant activity of honeys from the state of Rio Grande do Sul, Brazil. Food Science and Technology, 65: 333-340. 10.1016/j.1wt.2015.08.018.

Chanchao, C. (2009). Antimicrobial activity by Trigona laeviceps (Stingless Bee) honey from Thailand. Pakistan Journal of Medical Sciences, 25: 364-369.

Chua, L. S., Rahaman, N. L. A., Adnan, N. A. \& Tan, T. T. E. (2013). Antioxidant Activity of Three Honey Samples in relation with Their Biochemical Components. Journal of Analytical Methods in Chemistry, 2013: 1-8. 10.1155/2013/313798.

CLSI - Clinical \& Laboratory Standards Institute. (2003). Methods for dilution antimicrobial susceptibility tests for bacteria that grow aerobically. NCCLS docment M7-A6, v.23, 88p.

Cruz, C. B. N., Pieri, F. A., Carvalho-Zilse, G. A., Orlandi, P. P., Nunes-Silva, C. G. \& Leomil, L. (2014). Antimicrobial activity of honeys from two stingless honeybee species and Apis mellifera (Hymenoptera: Apidae) against pathogenic microorganisms. Acta Amazonica, 44: 287-290. 10.1590/S004459672014000200015

Freitas, B. M. (2003). Meliponíneos. Universidade Federal do Ceará. Centro de Ciências Agrárias. http://www.abelhas.ufc.br/documentos/meliponineos.pdf.

Gima, M. B. S., Côrrea, J. S., Correa, M. S. O., Neto. J. G. H., Teixeira, J. P., Marques; R. B., Santarem, M. G. F. \& Azevedo, A. A. P. (2020). Microbiological characteristics and resistance profile of micro-organisms causing hospital infections in an ICU for pediatric patients in a hospital references in Amazon infectology. Brazilian Journal of Health Review, 3: 8663-8678. 10.34119/bjhrv3n4-114.

Gonçalves, A. L., Filho, A. A. \& Menezes. H. (2005). Atividade antimicrobiana do mel da abelha nativa sem ferrão Nannotrigona testaceicornis (Hymenoptera: Apidae, Meliponini). Arquivos do Instituto Biológico, 72: 455-459.

Kollef, M. H (2003). Antibiotics for the critically ill: more than just selecting appropriate initial therapy. Crit Care. 2; 17 (3):146.

Kivrak, S., Kivrak, I. (2017). Assessment of phenolic profile of Turkish honeys. International Journal of Food Properties, 20: 864-876. $10.1080 / 10942912.2016 .1188307$

Lira, A. F., Sousa, J. P. L. M., Lorenzon, M. C. A., Vianna, C. A. F. J. \& Castro, R. N. (2014). Estudo comparativo do mel de Apis mellifera com méis de meliponíneos. Acta Veterinaria Brasilica, 8: 169-178.

Michener, C. D. (1974). The Social Behavior of the Bees. Massachussets, Cambridge University: Havard University. 404p.

Miorin, P. L., Levy Junior, N. C., Custodio, A. R., Bretz, W. A. \& Marcucci, M. C. (2003). Antibacterial activity of honey and propolis from Apis mellifera and Tetragonisca angustula against Staphylococcus aureus. Journal of Applied Microbiology, 95: 913-920. 10.1046/j.1365-2672.2003.02050.x.

Molan, P. C. (2001). Potential of honey in the treatment of wounds and burns. American Journal of Clinical Dermatology, 2: 13-19. 10.2165/00128071200102010-00003.

Moreira, D. L., Suzana, G. L., Gonçalves, J. L., Wigg, M. D. \& Leitão, G. G. (2005). Antioxidant and antiviral properties of Pseudopiptadenia contorta (Leguminosae) and of quebracho (Schinopsis sp.) extracts. Química Nova, 28: 421-425. 10.1590/S0100-40422005000300011.

Olaitan, P. B., Adeleke, O. E. \& OlaI, O. (2007). Honey: a reservoir for microorganisms and an inhibitory agent for microbes. African Health Sciences, 7: 159-165. 10.5555/afhs.2007.7.3.159.

Oliveira, P. S.; Müller, R. C. S.\& Dantas, K. G. F. (2012). Phenolic acids, flavonoids and antioxidant activity in honey of Melipona fasciculata, M. flavolineata (Apidae, Meliponini) and Apis mellifera (Apidae, Apini) from the Amazon. Quimica Nova, 35: 9, 1728-1732.

Pereira, A. S., Shitsuka, D. M., Parreira, F. J., \& Shitsuka, R. (2018). Metodologia da Pesquisa Cientifica [e-book]. UFSM. https://repositorio.ufsm.br/bitstream/handle.

Rodrigues A. S. (2006). Até quando o etnoconhecimento sobre as abelhas sem ferrão (Hymenoptera, Apidae, Meliponinae) será transmitido entre gerações pelos índios Guarani M’byá da Aldeia Morro da Saudade, localizada na cidade de São Paulo, Estado de São Paulo, Brasil? Sitientibus Série Ciências Biológicas, 6:343-350.

Silva, M. L. C., Costa, R. C., Santana, A. S., Koblitz, M. G. B.(2010). Compostos fenólicos, carotenóides e atividade antioxidante em produtos vegetais. Semina: Ciências Agrárias, v. 31, n. 3, p. 669-681

Silveira, F. A., Melo, G. A. R. \& Almeida, E. A. B. (2002). Abelhas brasileiras: Sistemática e identificação. Editora Composição e Arte, Minas Gerais, Brasil. $1^{a}$ edição, 253p.

Singleton, V. L., Orthofer, R. \& Lamuela-Raventós, R. M. (1999). Analysis of total phenols and other oxidation substrates and antioxidants by means of FolinCiocalteu reagent. Methods in Enzymology, 299: 152-178. 10.1016/S0076-6879(99)99017-1.

Vaerewijck, M. J. M., De Vos, P., Lebbe, L., Scheldeman, P., Hote, B. \& Heyndrickx, M. (2001). Ocurrence of Bacillus sporothermodurans and other aerobic spore-forming species in feed concentrate for dairy cattle. Journal of Applied Microbiology, 91: 1074-1084. 10.1046/j.1365-2672.2001.01477. x. 